\title{
Abschlussbericht zum DBU-Projekt: „Feinstaubsensor zur Schadstoffminderung in einer Regelung des Abbrandverhaltens biogener Festbrennstoffe"
}

\author{
Thorsten Wagner ${ }^{1}$, Alexander Weiß², Sabrina Eichenauer ${ }^{3}$, Ernst A. Stadlbauer ${ }^{3}$ Claus-Dieter Kohl \\ 1 Universität Paderborn, Warburger Straße 100, D-33098 Paderborn, \\ Tel.-Nr.: +49 525160 2486, thorsten.wagner@upb.de \\ 2 Institut für Angewandte Physik, JLU Gießen, Heinrich-Buff-Ring 16, D-35392 Gießen, \\ Tel.: +49 641 9933483, claus-dieter.kohl@ap.physik.uni-giessen.de \\ 3 Zentrum für Energie- und Umweltsystemtechnik, THM Mittelhessen, Wiesenstr. 14 D-35390 Gießen, \\ Tel.: +49 641 3092461, ernst.a.stadlbauer@zeuus.thm.de
}

\section{Zusammenfassung}

Das durch den Klimawandel verstärkte Interesse an $\mathrm{CO}_{2}$-neutralen Energieträgern führt zu einer zunehmenden Nutzung holzbeschickter Kleinfeuerungsanlagen (KFA). Der Beitrag der meist ungeregelt ablaufenden Verbrennungsprozesse zur Luftschadstoffbelastung, vor allem durch Feinstaub, ist überproportional hoch. Im Folgenden werden Ansätze vorgestellt, welche Möglichkeiten insbesondere im Bereich der Regelung solcher Anlagen bestehen, um eine Emissionsminderung herbeizuführen. Insbesondere wird auf das im Rahmen des Projektes erstellte Funktionsmuster zur kontinuierlichen Messung des Feinstaubs häuslicher Holzfeuerungsanlagen auf Basis eines elektrischen Verfahrens (Impedanzmessung) eingegangen.

Keywords: Feinstaubsensor, Kleinfeuerungsanlagen, Impedanzmessung, Abbrandregelung

\section{Einleitung}

Das durch den Klimawandel verstärkte Interesse $\mathrm{CO}_{2}$-neutraler Energieträger führt zu einer zunehmenden Nutzung holzbeschickter Kleinfeuerungsanlagen (KFA). Neben der besseren $\mathrm{CO}_{2}$-Billanz im Vergleich zu anderen Energieträgern wie Öl oder Gas ist Holz auch kostenmäßig selbst nach dem drastischen Preisverfall für Heizöl in 2015 (Heizöl: ca. 5,3 Cent/kWh [1]; Scheitholz: ca. 5,83 Cent/kWh [2]) konkurrenzfähig. Die Nutzung des Brennstoffes Holz zeigt sich auch in der Entwicklung der Zahl der in Deutschland vorhandenen Anlagen (siehe Abb.1). Bereits 2005 stellten sie bereits mehr als 40\% (14 Mio. Anlagen [3]) der kleinen und mittleren Feuerungsanlagen. Neben dem positiven Effekt der Einsparung von $\mathrm{CO}_{2}$ besitzen viele der bestehenden Anlagen jedoch auch einige Nachteile. Die Möglichkeiten zur Einstellung der richtigen Stöchiometrie im Verbrennungsprozess (typischerweise unterstöchiometrische Verbrennung) sind bei privaten und kleingewerblichen Anlagen wie den typischerweise eingesetzten Scheitholzvergaser mit kombinierter Vergasungs- und Verbrennungszone bauartbedingt begrenzt; insbesondere da dort keine geeigneten Messgrößen erfasst werden. Die Folge ist eine erhöhte Produktion an Stickoxiden, Ruß, Teer (organische Kondensate) sowie polyzyklischer aromatischer Kohlenwasserstoffe (PAK). Neben den gasförmigen Emissionen stellen im speziellen die Feinstäube dabei ein erhöhtes gesundheitliches Risiko für Mensch und Umwelt dar.

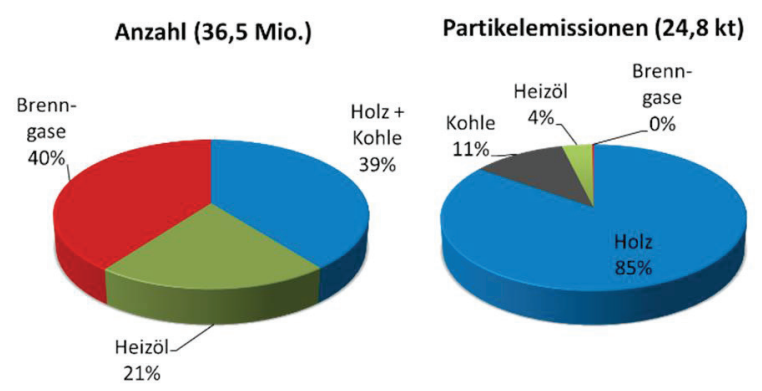

Abb.. 1: Kleinfeuerungsanlagen in Haushalten und Gewerbe, Handel, Dienstleistungen sowie Partikelemissionen in Deutschland (2005) [3].

Auf Basis ihrer größenabhängigen Wirksamkeit findet eine Klassifizierung nach Abscheideverhalten im menschlichen Körper statt. Teilchen der Klasse PM10 (Partikel $d<10 \mu \mathrm{m}$ ) gelangen bis in die Nasenhöhle. Teilchen der

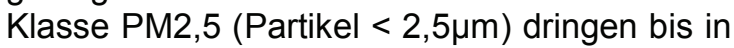


die Bronchien und Lungenbläschen und ultrafeine Stäube $(<0,1 \mu \mathrm{m})$ sogar bis in das Lungengewebe und den Blutkreislauf vor. Die Schäden je nach Größe und Eindringtiefe können von Schleimhautreizungen bis zu lokalen Entzündungen der Lungenbläschen reichen. Bei dieser Klassifizierung wird die chemische Identität der Partikel allerdings vernachlässigt. Diese ist jedoch nach aktueller Meinung für die Einschätzung der Toxizität mindestens ebenso relevant wie die Größe der Partikel. Eine grobe Einteilung kann dabei in überwiegend aus Kohlenstoff bestehenden Ruß (black carbon, BC), mineralische Salze und kondensierbare organische Verbindungen (COC, auch als Teer bezeichnet) erfolgen. Neben diesen primären, d.h. direkt bei der Verbrennung entstehenden Aerosolen, können auch sekundäre organische Aerosole aus den flüchtigen organischen Verbindungen (VOCs), Ammoniak, Kohlenmonoxid sowie Stickoxiden entstehen [4].

Eine Möglichkeit, der Entstehung von Feinstaub entgegenzuwirken, ist eine Trennung der Zonen zur Gaserzeugung und Verbrennung durch eine Reduktionszone. Dies erlaubt prinzipiell den Eingriff in den Prozess durch eine Regelung zur Optimierung der Abbrandbedingungen. Dazu werden jedoch geeignete Messglieder in Form von Sensoren im speziellen für die Feinstaubkomponenten benötigt, die unter den sehr rauen Bedingungen arbeiten. Inhalt des vorgestellten Projektvorhabens war die Entwicklung und Erprobung von Komponenten eines Funktionsmusters für eine kontinuierliche in-situ Feinstaubmessung von Kleinfeuerungsanlagen. Ziel ist, die Fehleranfälligkeit im Zusammenwirken von Brennstoff, Feuerstätte und Nutzer durch Integration neuartiger Sensortechnik zur Kontrolle und Steuerung des Abbrand-verhaltens zu minimieren. Im vorgestellten Projekt wurde ein Funktionsmuster zur kontinuierlichen Messung des Feinstaubs häuslicher Holzfeuerungsanlagen auf Basis eines elektrischen Verfahrens (Impedanz-messung) erstellt und optimiert.

\section{Partikelbildung Holzverbrennung}

In der Literatur sind fünf Hauptpfade zur Partikelbildung bei der Holzverbrennung beschrieben [5]. Zentral ist dabei die Verdampfung der anorganischen Holzinhaltsstoffe (Metallkationen wie $\mathrm{K}, \mathrm{Na}, \mathrm{Ca}, \mathrm{Mg}$, Schwermetalle, Ammoniak) und Anionen (Chlorid, Sulfat) begleitet vom Mitreißen von Partikeln aus der Asche, Neubildung und Umlagerung in Gasphasenreaktionen und bei Kondensationsprozessen (z.B. $\mathrm{CaO}, \mathrm{SiO}_{2}$, $\mathrm{Al}_{2} \mathrm{O}_{3} ; \mathrm{KCl}, \mathrm{K}_{2} \mathrm{SO}_{4}, \mathrm{NaCl}, \mathrm{NH}_{4} \mathrm{Cl}$ ). Darüber hinaus entstehen bei unvollständigem
Ausbrand in-situ niedermolekulare kohlenstoffhaltige Verbindungen als Fragmente pyrolytischer Prozesse von Cellulose, Hemicellulose und Lignin. Diese Stoffgruppen stellen die makromolekularen Hauptbestandteile holzartiger Materialien dar [6]. Unter diesen organischen Abbauprodukten ist Zersetzungsprodukten wie z.B. Ruß und insbesondere kondensierten aromatischen Ringsystemen, den sogenannten polyzyklischen aromatischen Kohlenwasser-stoffen (PAK), verstärkte Aufmerksamkeit zu widmen. Angelagert an mineralische Komponenten oder elementaren Kohlenstoff (elemental carbon, EC) im Submikrobereich prägt diese Stoffklasse im besonderen Maße das Toxizitätspotential von Partikelemissionen aus häuslicher Holzfeuerung [7]. Analytisch werden PAK bei der Bestimmung des Summenparameters Organic Carbon (organischer Kohlenstoff, OC) mit erfasst. Hingegen ermöglicht eine Einzelanalyse eine stoffbezogene Risikobewertung.

Spezifische Wechselwirkungen einzelner Inhaltsstoffe und Dosis-Wirkungs-Beziehungen sind heute noch Gegenstand intensiver weltweiter Forschung $[8,9]$. Fest steht, dass das Gefährdungspotenzial neben der Größenklasse $(\mathrm{GK}<1 \mu \mathrm{m})$ und Konzentration vor allem durch die chemische Natur geprägt wird.

Das Gesundheitsrisiko der verschiedenen Gruppen von Inhaltsstoffen der Feinstaubemissionen aus Biomassefeuerungen steigt von anorganischen Salzen $\left(\mathrm{mg} / \mathrm{Nm}^{3}\right)$ über organischen Kohlenstoff, elementaren Kohlenstoff jeweils in $\mathrm{mg} / \mathrm{Nm}^{3}$, hin zu den besonders kritischen polyzyklischen aromatischen Kohlenwasserstoffen, PAK $\left(\mathrm{ng} / \mathrm{Nm}^{3}\right)$ an $[10,11]$.

Eine ideale Sensorik sollte also neben der Größenverteilung am besten auch eine Klassifizierung der Partikel hinsichtlich der chemischen Zusammensetzung erlauben.

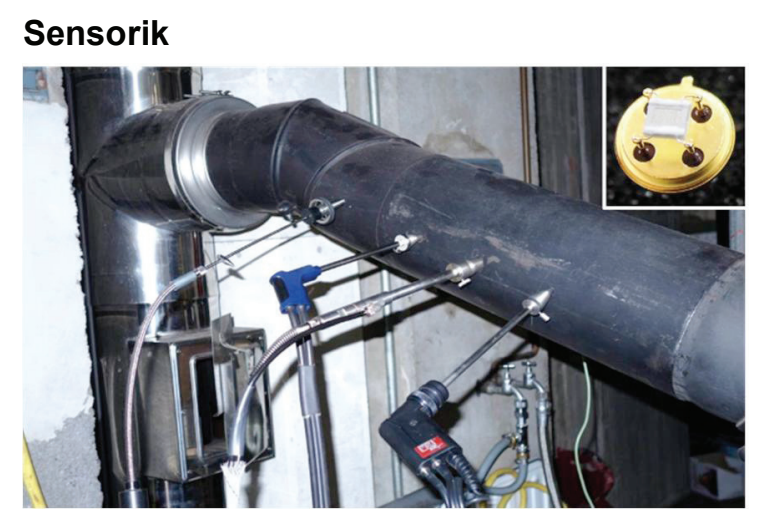

Abb.. 2: Messanordnung zur Beladung der Sensorsubstrate (inset) und gleichzeitiger Partikelmessung mit Referenzgeräten. 
Im Gegensatz zu Großfeuerungsanlagen oder der Steuerung von Verbrennungsmotoren (Lambdasonde), ist eine kontinuierliche Sensorik in holzbefeuerten KFA kaum verbreitet. Wenn vorhanden, handelt es sich hauptsächlich um temperatursensitive Elemente zur Leistungsregelung. Entweder steuern diese mechanisch (Bimetall, Thermostat) die Zuluft oder sie regulieren die Brennstoffmenge in modernen Holzpelletanlagen. Neben einer Messung der Kesseltemperatur bei wasserführenden Systemen können auch Thermoelemente im Bereich des Brennraums genutzt werden. Eher selten werden Lambdasonden eingesetzt, um die Verbrennungsluftzufuhr zu steuern. Die im Wesentlichen an KFA genutzte Messtechnik bezieht sich auf die gesetzlich vorgeschriebenen regelmäßigen Messungen zur Überwachung der Grenzwerteinhaltung. Im Gegensatz zu kontinuierlichen Messungen kann durch diese Stichproben jedoch keine Regelung der Verbrennung erfolgen, sondern lediglich grobe Falscheinstellungen und Defekte aufgespürt werden (z.B. Undichtigkeiten etc.). Dabei werden durch den Schornsteinfeger neben Staub und $\mathrm{CO}$, auch die Temperatur, der Sauerstoffgehalt und die Druckdifferenz zur Bewertung des Betriebszustandes in einer 15-minütigen Messung festgestellt. $\mathrm{Zu}$ den verwendeten Messverfahren bei der Staubmessung zählen optische (Streulicht, Photoemission), elektrostatische und vor allem gravimetrische Verfahren. Letztere sind im einfachsten Fall Filterhülsen, die nach der Probenahme gewogen werden, oder kontinuierlich anzeigende Wiegeverfahren, wie z.B. Quarzmikroschwinger oder makroskopische Schwingelemente. Die gasförmigen Messgrößen $\left(\mathrm{CO}, \mathrm{O}_{2}, \mathrm{NO}_{\mathrm{x}}\right)$ werden bei allen gängigen Geräten mit Hilfe von elektrochemischen Zellen ermittelt. Die Temperaturmessung erfolgt mit Thermoelementen (Typ K).Die für die Messung nach 1. BImSchV zugelassenen Geräte weisen bzgl. des Staubgehalts unabhängig von ihrem Messprinzip eine hohe Messungenauigkeit von $30 \%$ bis $50 \%$ [12] auf.

Um Sensorik zur Regelung der Betriebsparameter in KFA einsetzen zu können, muss das Messverfahren zum einen kontinuierlich arbeiten und zum anderen durch die technische Umsetzung den rauen Umgebungs-bedingungen in der Feuerungsanlage bzw. der Abluftrohre standhalten. Im Gegensatz zur Erkennung z.B. eines Filterschadens im KFZ (relevante Zeit: Stunden, Tage), muss ein Sensor zur Regelung einer Holzverbrennung auf der Zeitskala unterhalb einer Minute arbeiten. Eine schnelle Reaktion und die Detektion kleiner
Staubmengen sind daher notwendig. Dazu wurde im Projekt eine kapazitive Auswertung des Sensors genutzt. Auf und zwischen den Elektroden abgeschiedene Partikel führen dabei auf Grund ihrer von Luft verschiedenen Permitivität zu einer messbaren Änderung der Kapazität, noch bevor ein leitfähiger Pfad entsteht. Während bei resistiver Messung - eine gleichmäßige Bedeckung mit hinreichend kleinen Staubpartikeln vorausgesetzt Elektroden-abstand und -größe prinzipiell keine Rolle spielen, haben sie bei kapazitiven Verfahren einen starken Einfluss auf Grundkapazität und Sensitivität. Eine Anpassung der Struktur bietet also die Möglichkeit, den Sensor auf eine spezielle Anwendung hin weiter zu optimieren.

\section{Impedanzmessungen}

Der vorliegende Ansatz zur in-situ Bestimmung des Feinstaubs verwendet ein keramisches Sensorsubstrat mit Elektroden in Interdigitalstruktur und integrierter Heizung. Dieses Substrat wurde mit Hilfe eines speziell konstruierten Sensorträgers im Abgasstrom positioniert (siehe Abb.2). Obwohl diese Methode prinzipiell in-situ durchgeführt werden kann, wurde im Rahmen des Projektes jedoch zunächst die ex-situ Charakterisierung gewählt, um im ersten Schritt etwaige Quereinflüsse zu minimieren und ein prinzipielles Verständnis für das Sensorverhalten zu entwickeln. Im zweiten Schritt können dann Verfahren zur Kompensation dieser Einflüsse integriert werden. Nach vorher festgelegter Depositionszeit wurden die Substrate deshalb aus dem Abgasstrom entnommen und in einer eigens entworfenen Messkammer (siehe Abb.3) charakterisiert. Die Messkammer ermöglicht die Messung in definierter Atmosphäre und schirmt die Sensoren zusätzlich gegen elektromagnetische Störfelder ab. Ein Gas-Mischsystem erzeugt für die Messungen konstante Bedingungen in der Messkammer.

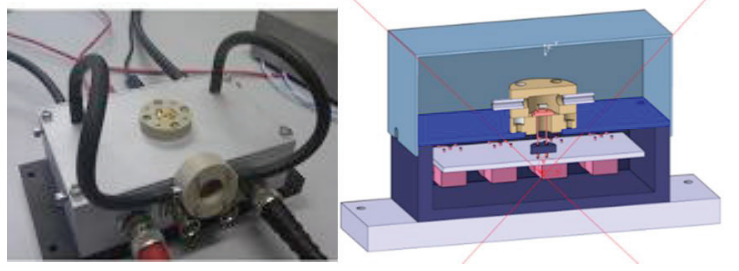
Abb.. 3: Messkammer mit abgenommener Schirmung und geöffnetem Sensorraum (links) und in einem CAD-Schnitt (rechts).

Die eigentliche Messung erfolgte mit einem Solartron SI1260 Impedanz-spektrometer und einem Transimpedanz-verstärker (Chelsea Dielectric Interface) zur Messbereichserweiterung. Die Amplitude der verwendeten 
anregenden Sinusspannung betrug $500 \mathrm{mV}$. Der Staub lagert sich während der Deposition zwischen den Elektroden ab, wo seine dielektrischen Eigenschaften zu einer messbaren Kapazitätsänderung der Elektroden führen. Nach einer Messung wird durch Anlegen einer Heizspannung das Sensorsubstrat aufgeheizt und somit freigebrannt. Der Energieeintrag während dieser Freibrennphase liefert zusätzliche Messdaten, die prinzipiell Rückschlüsse auf die Zusammensetzung des Staubes zulassen sollten (Inhalt weiterer Untersuchungen). Somit stehen auf nur einem Sensor zwei voneinander unabhängige Messgrößen zur Verfügung. Dadurch lässt sich unter Einbeziehung angepasster Auswertealgorithmen die Staubbelastung sicherer und genauer bestimmen. Die Ermittlung des Staubgehalts erfolgt aus dem kapazitiven Anteil der Impedanz. Die Kapazität der Sensoren wurde jeweils einzeln im Neuzustand vermessen, da es zu geringen herstellungsbedingten Variationen der Leerkapazität (CO) kommen kann.

Die Berechnung der Kapazität erfolgte nach folgender Formel (1):

$$
\operatorname{Re}(C)=\frac{-\operatorname{Im}(Z)}{\omega\left(\operatorname{Re}(Z)^{2}+\operatorname{Im}(Z)^{2}\right)}
$$

Dieses Vorgehen erlaubt eine Betrachtung der Kapazität über den gesamten gemessenen Frequenzbereich und würde im idealisierten Fall ein frequenzunabhängiges Verhalten liefern.

Auf Basis von Voruntersuchungen wurde für Vergleichsmessungen eine Messfrequenz von $1,6^{*} 10^{4} \mathrm{~Hz}$ ausgewählt.

Wiederholende Messungen zu verschiedenen Zeiten zeigten Abweichungen von nur 0,02 \%. Variationen der Feuchte im relevanten Bereich zwischen $0 \%$ und $30 \%$ r.h. ergeben Signalunterschiede von $0,03 \%$.

\section{Ergebnisse}

Im Folgenden sind exemplarisch die Ergebnisse einer Messreihe im Betrieb einer Testanlage mit Holzpellets (siehe Abb.4) im Vergleich mit einem Referenzgerät gezeigt. Weitere Messreihen und eine ausführlicherer Diskussion finden sich im Abschlussbericht des Projektes [13]. Für die im Folgenden gezeigten Messpunkte wurden jeweils 2 nominell identische Sensoren sowie das Referenzgerät (Wöhler SM96, zugelassen nach 1.BImSchV) verwendet. Die Platzierung der zwei gleichen Sensoren erfolgte unmittelbar nebeneinander auf dem Sensorträger. Damit sollte die Messunsicherheit des Systems bestimmt werden.

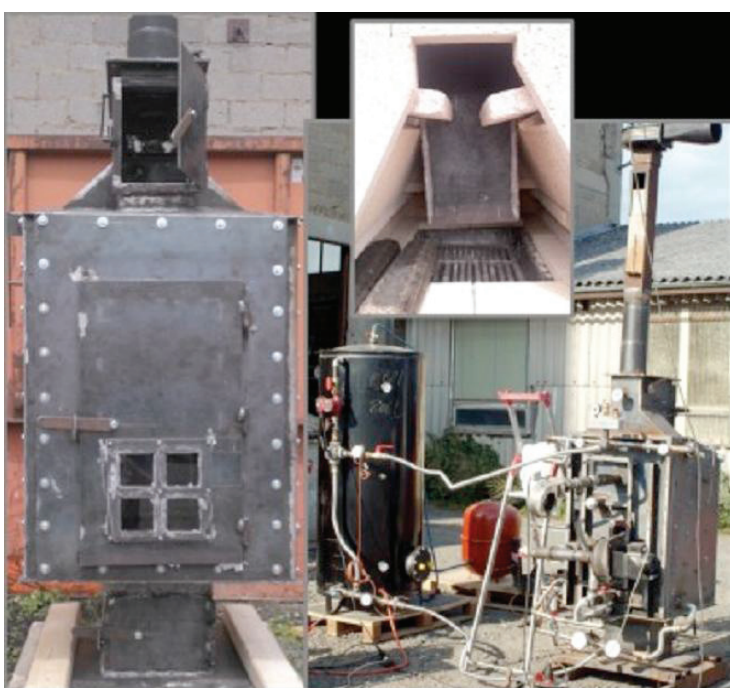

Abb. 4: Prototyp einer Kleinfeuerungsanlage der Fa. Strohal; Experimentelle Anordnung mit variablen Einsätzen.

Die Abbildung 5 zeigt die Sensorreaktion von 6 aufeinanderfolgenden Messungen (Beladung je 15 Minuten), wobei die Werte der Impedanzsensoren gemittelt wurden und ihre jeweilige Abweichung voneinander als Fehlerbalken angegeben ist. Die durchschnittliche Abweichung beträgt $30 \%$ der Signalhöhe, die maximale Abweichung beträgt 66 \% (Messung \#1). Die Korrelation der Daten mit dem Referenzgerät ist deutlich zu erkennen.
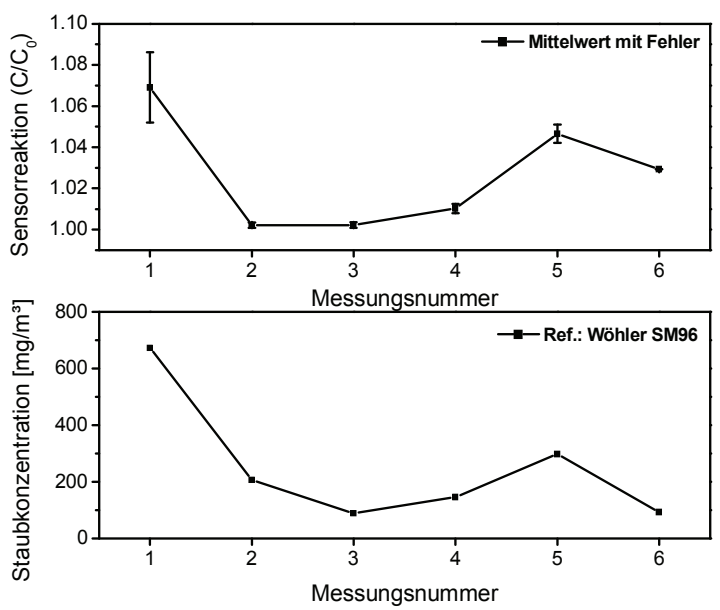

Abb. 5: Messreihe bei Fa. Strohal mit firmeneigenem Prototyp: Vergleich der Kapazitätsänderung zweier gleichartiger Sensoren (angegeben als Mittelwert mit Fehlerbalken für die Abweichung) mit den Ergebnissen des Referenzgerätes Wöhler SM96.

Wie die Referenzmessung zeigt, liegt die Staubkonzentrationen dieser Versuchsreihe relativ hoch und durchweg über den Grenzwerten. Auf dem Substrat wird so viel Material abgeschieden, dass die Perkolationsschwelle bereits überschritten wird. Eine 
wesentliche Verkürzung der Messzeit ist somit möglich und wäre im Sinne einer Regelungstechnik auch notwendig. Die Sensoren konnten jeweils durch Freibrennen regeneriert werden. Weiterhin konnte im Verlauf des Projektes gezeigt werden, dass auch geringere Massendepositionen mit einer Sensorreaktion von nur $0,2 \%$ noch klar vom Rauschen trennbar sind.

\section{Ausblick}

In dem abgeschlossenen Projekt konnte unter anderem die prinzipielle Funktion eines impedanzbasierten Ansatzes zur Messung von Partikelkonzentrationen im Gasstrom gezeigt werden. Die erzielten Resultate lassen darauf schließen, dass für eine Regelung notwendige Messzeiten erreichbar sind. Zukünftig muss allerdings noch die Notwendigkeit der Kompensation bezüglich variabler Temperatur, veränderlichem Druck bzw. unterschiedlicher Stärke des Abgasstromes während der verschiedenen Abbrandphasen näher untersucht werden.

Weiterhin können zusätzliche Signale, die bei der Messung anfallen, zur genaueren Bestimmung der chemischen Natur der Partikel herangezogen werden, z.B. die unterschiedlichen Leistung beim Freibrennen der Sensorsubstrate.

Abschließend muss außerdem eine Übertragung der Kapazitätsmessung vom Labormaßstab auf eine kompakte und kostengünstigere Elektronik stattfinden. Weiteres Optimierungspotential besteht zudem im Aufbau des verwendeten Substrats.

\section{Danksagung}

Wir danken Bernd Vollmer und Hans-Eberhard Kopp (Schornsteinfegerakademie Dülmen) sowie Ulrich Strohal und Jochen Fey (Strohal Anlagenbau, Staufenberg) für Ihre fachliche Unterstützung und die Bereitstellung der Feuerungsanlagen. Weiterer Dank gilt der Deutschen Bundesstiftung Umwelt für die Finanzierung des Projekts.

\section{Literaturnachweis}

[1] Quelle depi.de; abgerufen Februar 2016

[2] Quelle tfz.bayern.de; abgerufen Februar 2016

[3] M. Struschka, D. Kilgus, M. Springmann, G. Baumbach, Effiziente Bereitstellung aktueller Emissionsdaten für die Luftreinhaltung, Umweltbundesamt, Texte 44/2008

[4] T. Nussbaumer, Emissionsfaktoren von Holzfeuerungen und Klimaeffekt von Aerosolen aus der Biomasse-Verbrennung, 11. Holzenergie-Symposium, Zürich, 17. (2010)

[5] M. Oser, T. Nussbaumer, P. Müller, M. Mohr, R. Figi, Grundlagen der Aerosolbildung in Holzfeuerungen: Beeinflussungen der Partikelemissionen durch Primärmaßnahmen und Konzept für eine partikelarme automatische
Holzfeuerung (Low-Particle-Feuerung).

Bundesamt für Energie, Bundesamt für Umwelt, Wald und Landwirtschaft, Bern (2003)

[6] G. Baumbach, Identifizierung von Holzfeuerungsabgasen in Wohngebieten.

Präsentation, 5. Fachkolloquium

Feuerstätten/Abgasanlagen

Verbrennungssysteme, Stuttgart, 30. April 2013

[7] Umweltbundesamt: Hintergrundpapier Feinstaubbelastung in Deutschland. Pressestelle UBA, Mai 2009

[8] B. Bellmann, Saubere Biomasseverbrennung in Zentralheizungsanlagen: Bestimmung der Partikelgrößen, Probenahme und physikochemisch-toxikologische Charakterisierung, Schlussbericht Verbundprojekt, EraNet Bioenergy "BiomassPM", FZK: 22011406 (2008)

[9] WHO (Hrsg.): Air Quality Guidelines. Global Update 2005. Particulate matter, ozone, nitrogen dioxide and sulfur dioxide. World Health Organization, Regional Office for Europe. Kopenhagen(2006)

[10] T. Nussbaumer, N. Klippel, M. Oser,, Health relevance of aerosols from biomass combustion in comparison to Diesel soot indicated by the cytotoxicity on lung cells, Aerosols in Biomass Combustion, Series Thermal Biomass Utilization, Graz University of Technology, Seiten 45-54, 6 (2005)

[11] UMEG: Emissionsmessungen an Holzfeuerungen im Regelungsbereich der 1. BImSchV. Feldmessprogramm in BadenWürttemberg, Heizperiode 2002 / 2003. UMEG Zentrum für Umweltmessungen, Umwelterhebungen und Gerätesicherheit Baden-Württemberg, Bericht Nr. 43-2 18/03. Karlsruhe (2003)

[12] VDI-Richtlinie 4206 Blatt 2: Mindestanforderungen und Prüfpläne für Messgeräte zur Überwachung der Emissionen an Kleinfeuerungsanlagen. Düsseldorf, VDI 2011

[13] E. Stadlbauer, S. Eichenauer, A. Weiß, M. Bauer, C.-D. Kohl, Feinstaubsensor zur Schadstoffminderung in einer Regelung des Abbrandverhaltens biogener Feststoffe, Abschlussbericht über ein Entwicklungsprojekt, gefördert unter dem Az: 30960-21/0 von der Deutschen Bundesstiftung Umwelt (2014) 